\title{
Malignant right coronary artery in an athlete: Evidence-less based medicine?
}

\author{
Authors: Raja EF Raja Shariff ${ }^{\mathrm{A}}$ and Sazzli S Kasim ${ }^{\mathrm{A}}$
}

\begin{abstract}
A 31-year-old man presented with central chest heaviness. He was a smoker of 15 pack-years, but otherwise had no other comorbidities. He was also a professional footballer. There was no family history of sudden cardiac deaths of note. In view of a low to intermediate pre-test probability for coronary artery disease (CAD), computed tomography coronary angiography (CTCA) was performed, revealing an anomalous, malignant right coronary artery (RCA), originating from the left main coronary stem. Malignant RCAs are rare, and the majority of patients remain asymptomatic. However, malignant RCAs have been associated with both myocardial infarctions and sudden cardiac deaths, which has led to difficulty in deciding on whether a 'watchful waiting' approach or more proactive approach should be adopted. Unfortunately, there remains a lack of evidence to help guide treatment decisions. Furthermore, there are no known guidelines on managing coronary anomalies in athletes, such as the case presented. As the majority of national guidelines have largely recommended CTCA as first-line investigation in patients with low to intermediate risk of CAD with chest pain, incidental finding of coronary anomalies will become more common, urging the need for guidelines to help with directing management in such cases.
\end{abstract}

KEYWORDS: Cardiac computed tomography, coronary artery disease, congenital heart disease

DOI: 10.7861/clinmed.2019-0501

\section{Case presentation}

A 31-year-old man presented to our emergency department with sudden onset of central chest heaviness, lasting approximately 20 minutes. This was associated with dyspnoea and diaphoresis. He was a smoker of 15 pack-years, but otherwise had no other comorbidities. He was also a professional footballer for his local team, and had regular training sessions weekly but had been refraining from participating in matches over the last 6 months due to intermittent chest discomfort of similar nature. There was no family history of sudden cardiac deaths of note. His vitals

Authors: ${ }^{\text {A }}$ cardiology fellow and clinical lecturer, Universiti Teknologi MARA, Sungai Buloh, Malaysia on arrival were unremarkable, with a heart rate of 56 beats per minute, blood pressure of $110 / 76 \mathrm{mmHg}$ and oxygen saturation of $98 \%$ on room air. Clinical examination was unremarkable, with no evidence of any cardiac murmurs. High-sensitivity troponin 3-hours apart were within normal range and electrocardiography (ECG) was unremarkable. In view of a low to intermediate pretest probability for coronary artery disease (CAD), a computed tomography coronary angiography (CTCA) was performed (Fig 1).

\section{Diagnosis}

The CTCA demonstrated an anomalous origin of the right coronary artery (RCA), originating from the left main coronary stem before taking an inter-arterial course between both the aorta and the pulmonary artery. This is commonly known as a 'malignant RCA', an uncommon variant of RCA anomalies.'

\section{Initial management and prognosis}

The patient was provided with essential information on the condition, including the rare risk of myocardial infarction and sudden cardiac death. However, initial investigations were reassuring, and as the Agatston score calculated was zero and there were no coronary plaques noted from the CTCA, the patient was reassured in the emergency department and allowed home following a 24-hour period of observation. He was given a chest pain clinic appointment within a week for further assessment.

\section{Case progression and outcome}

The patient was provided with information on the condition but reassured regarding the findings. We provided lifestyle advice and support for smoking cessation. The patient was also started on rosuvastatin following investigations revealing a total cholesterol level of $5.0 \mathrm{mmol} / \mathrm{L}$ and low-density lipoprotein of $3.4 \mathrm{mmol} / \mathrm{L}$. The patient remains well under our general cardiology followup with no further episodes of similar symptoms since his initial presentation.

\section{Discussion}

Malignant RCAs are rare, with an incidence of 0.17 to $1.2 \%$. Although the majority of patients remain asymptomatic during their lifetime, with most cases being incidentally discovered, malignant RCAs have been associated with both myocardial infarctions and sudden cardiac deaths, especially in young 


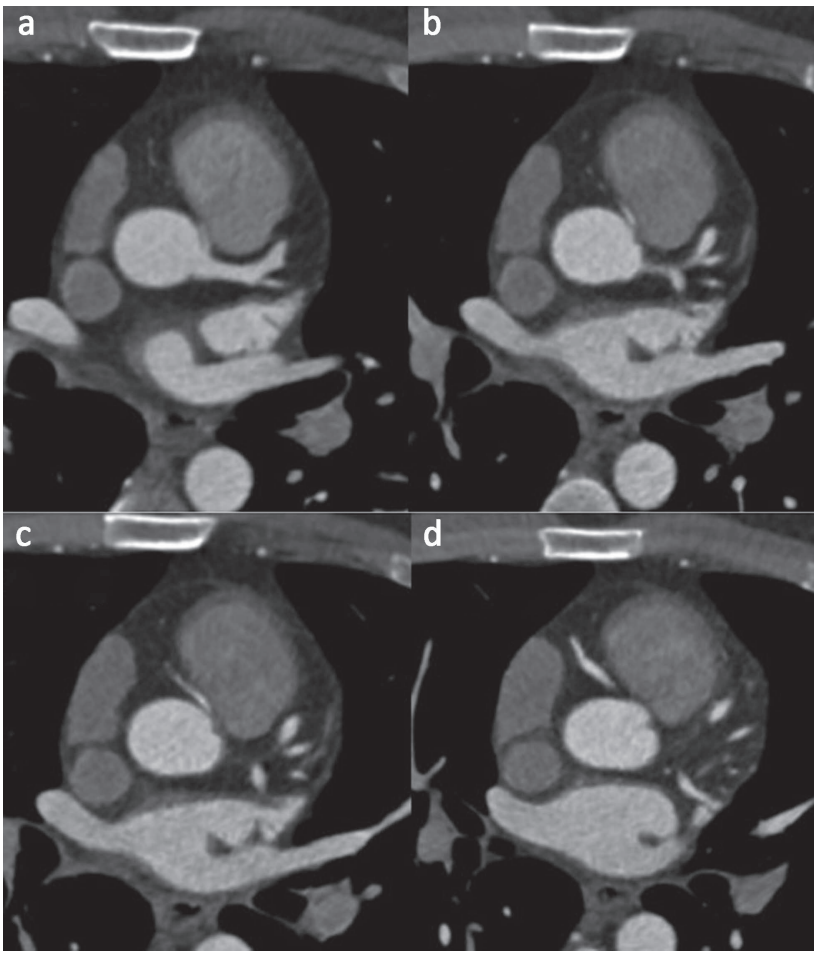

Fig 1. Computed tomography coronary angiography demonstrating an anomalous origin of the right coronary artery.

patients. Various contributing factors have been postulated, including mechanical compression between the aorta and pulmonary artery, the presence of valve like ridges, angulation of the artery as it courses and presence of a slit-like orifice. ${ }^{2}$

However, the incidence of sudden death remains very low and of note, only 10 cases have been reported in the literature up until present. This has led to difficulty in establishing evidence to support coronary intervention. At present, a 'watchful waiting' approach is reasonably adopted in most cases of asymptomatic, incidentally found anomalies, whereas revascularisation via surgical or percutaneous interventional means have been reported in the past. Surgical management (which includes unroofing and reimplantation, or coronary artery bypass grafting) has been reported to be superior versus percutaneous intervention based on poorer long-term outcomes due to risk of in-stent restenosis, thrombosis, stent fractures and coronary artery dissection. Furthermore, there have been reports on procedural-based complexities that contribute to procedural failures, including poor guiding catheter intubation and support. ${ }^{2}$

Unfortunately, there are no guidelines designed to tackle situations similar to our case. The patient had presented with typical history of angina, but remained relatively low risk in view of his age and lack of comorbidities. Furthermore, he was an athlete involved in high-intensity physical activity. Despite fearing a possible risk of prolonged ischaemia which may subsequently lead to worsening myocardial infarction or ventricular dysfunction, we had made the decision to tackle his major risk factors - both smoking and dyslipidaemia, which we felt greatly contributed to his current symptom-free state. In view of lack of evidence, we had decided against performing serial CTCA for monitoring but instead opted for regular clinic follow-ups to monitor symptoms. This was to avoid unnecessary psychological (anxiety) and physical (radiation) harm. ${ }^{3}$ An exercise stress test and 72-hour Holter monitoring were also performed to assess his exercise capacity and risk of arrythmia (in view of RCA involvement), respectively, which were unremarkable.

The majority of national guidelines have largely recommended CTCA as a first-line investigation in patients with low to intermediate risk of CAD with chest pain, such as in our case. ${ }^{4}$ This will provide the additional benefit of screening for coronary anomalies, which were often difficult to recognise through conventional means of stress testing in the past. However, the decision to intervene and methods of monitoring following discovery of coronary anomalies remains vague, with ongoing lack of evidence to help guide management.

\section{Summary}

Although rare, coronary anomalies can present a challenge for the modern clinician, especially in an era where CTCA has been adopted as an initial investigative tool for CAD. Learning points from the case include:

> understanding pre-test probability calculation and decision making on next best step in management when facing symptoms suggestive of CAD

> appreciating how novel imaging techniques provide additional benefits of screening for structural anomalies, which were often missed in the past

> appreciating the complexity in deciding for intervention in patients with malignant RCAs

> appreciating the lack of guidelines and evidence to direct care for athletes with coronary anomalies.

\section{Acknowledgements}

The authors would like to acknowledge Universiti Teknologi MARA (UiTM) for supporting the submission of this case study.

\section{References}

1 Gajjar K, Khurana A, Patil A, Noor A, Meng J. Incidentally diagnosed anomalous right coronary artery with an interarterial course presenting as chest pain. Cureus 2019;11:e5264.

2 Suryanarayana P, Lee JZ, Abidov A, Lotun K. Anomalous right coronary artery: case series and review of literature. Cardiovasc Revasc Med 2015;16:362-6.

3 Melgies J, Hamilton MC, Manghat NE. Computed tomographic coronary angiography - is it ready as a screening tool for coronary artery disease? Clin Med 2013;13:465-71.

4 Moss A, Williams M, Newby D, Nicol E. The updated NICE guidelines: Cardiac CT as 1st line test for coronary artery disease. Curr Cardiovasc Imaging Rep 2017;10:15.

Address for correspondence: Dr Raja EF Raja Shariff, Universiti Teknologi MARA, Jalan Hospital, 47000 Sungai Buloh, Selangor, Malaysia.

Email: rajaezman@gmail.com 